\title{
Exploring EQ-5D-Y-3L Experience-Based VAS Values Derived Among Adolescents
}

\author{
Mimmi Åström ${ }^{1,2,3}$ (1) $\cdot$ Ola Rolfson ${ }^{1,4}\left(\right.$ Kristina Burström $^{1,2}(\mathbb{D}$
}

Accepted: 12 December 2021 / Published online: 26 January 2022

(c) The Author(s) 2022

\begin{abstract}
Objectives The EQ-5D-Y-3L is a generic health-related quality of life (HRQoL) instrument, developed from the adult version to be suitable for children and adolescents aged 8-15 years. To derive values for the EQ-5D-Y-3L different valuation methods and perspectives have been applied. The aim of this study was to explore EQ-5D-Y-3L experience-based visual analogue scale (VAS) values derived among adolescents.

Methods Data were derived from a cross-sectional population survey administered via schools in 2014 to adolescents aged 13-18 years, in Sweden. Regression analyses were performed on individual data with the VAS value as dependent variable. Ordinary least-squares (OLS) and generalised linear models (GLM) were estimated with two dummy variables for each of the EQ-5D-Y-3L dimensions. Interaction variables were tested. One way of anchoring VAS at dead and full health by using the predicted values for worst and best health states defined by the EQ-5D-Y-3L descriptive system was explored.

Results Of the 243 possible health states in EQ-5D-Y-3L, 92 were reported by the 6,468 respondents. The largest decrements in VAS values were observed for the dimension 'feeling worried, sad or unhappy' followed by 'doing usual activities'. All models performed similarly in terms of monotonicity and goodness of fit but in terms of simplicity and understandability, the OLS main effect model was superior.

Conclusions We have explored experience-based VAS values for the EQ-5D-Y-3L derived among adolescents. The findings suggest that it is possible for adolescents to value their own health state using the VAS, which makes it possible to capture aspects that are important for young people.
\end{abstract}

\section{Key Points for Decision Makers}

It is desirable to derive information from the target group of young people, for whom decisions are made, on the importance of different health dimensions. There

Mimmi Åström

mimmi.astrom@ki.se is an ongoing debate regarding which method and what perspective to use when eliciting values for EQ-5D-Y-3L health states.

1 Health Outcomes and Economic Evaluation Research Group, Department of Learning, Informatics, Management and Ethics, Stockholm Centre for Healthcare Ethics, Karolinska Institutet, Tomtebodavägen 18 A, SE-171 77 Stockholm, Sweden

2 Equity and Health Policy Research Group, Department of Public Health Sciences, Karolinska Institutet, Stockholm, Sweden

3 Centre for Health Economics, Informatics and Health Services Research, Stockholm Health Care Services, Region Stockholm, Stockholm, Sweden

This study explored EQ-5D-Y-3L experience-based VAS values among adolescents and found that the mood dimension had the strongest association with the VAS value.

The findings suggest that it is possible for adolescents to value their own health state using the VAS, which makes it possible to capture aspects that were important for young people in health state valuation.

4 Department of Orthopaedics, Institute of Clinical Sciences, the Sahlgrenska Academy, University of Gothenburg, Gothenburg, Sweden 


\section{Introduction}

The EQ-5D is a widely used generic health-related quality of life (HRQoL) instrument, which defines health status in five dimensions and is used in population health studies, clinical studies, as well as in health technology assessment [1]. The youth version of the instrument is the EQ-5D-Y$3 \mathrm{~L}$, which was developed in 2009. It generates 243 health profiles or health states by combining the five dimensions and the three severity levels for each dimension $[2,3]$.

Health outcomes measures, such as the EQ-5D, are essential to combine length of life and HRQoL into qualityadjusted life years (QALYs), the outcome used in cost-utility analysis [4]. To enable this, a value, i.e., a QALY weight, for each of the health states needs to be obtained. In determining such values, the most used direct valuation methods for adults are the Time-Trade Off (TTO), Standard Gamble (SG), Visual Analogue Scale (VAS), Discrete Choice Experiment (DCE), and Best-Worst Scaling methods (BWS) [4-6].

When asking children and adolescents to value health states both DCE and BWS have previously been used, but the SG and TTO have been questioned [6,7]. When using the VAS as a valuation method, respondents are asked to record a value for health states on a scale commonly numbered $0-100$. The VAS has been emphasised as a simple and low-cost valuation method [8] that is easy to understand [4, 5]. However, when using the VAS as a valuation method, the choice is not made under uncertainty, but whether VAS is a choice-based method has been discussed $[4,5]$. The VAS method has been used previously to derive so-called experience-based VAS values for children aged 10-11 years [9]. For adults, the VAS method has been used in several studies to derive experience-based value sets [10-15]. When predicted VAS values do not correspond to the 0 (dead) - 1 (full health) QALY scale required for the purpose of economic evaluation rescaling is needed [4]. To rescale the VAS value to 0 (dead) and 1 (full health) several approaches have been suggested; for example, simply by asking the respondent to place 'dead' on the scale $[11,16,17]$. However, recent theoretical assumptions for anchoring the VAS at 'dead' have been challenged [16]. Instead of anchoring the VAS at 'dead' it has been suggested to use the value of the worst health state defined in the descriptive system of an instrument [16]. To use the VAS for cost-utility analyses has been discussed by Parkin \& Devlin [18], presenting arguments for and against its use in economic evaluation, as well as presenting shortcomings with the commonly used TTO and SG methods. However, the authors emphasise the need to carry out empirical research to challenge the dominant preconceptions against the use of the VAS in cost-utility analyses [18].

In addition to deciding which valuation method to use, there is an ongoing debate with regard to who should value health states. For adults, the two main directions are experience-based values, which are derived from people who are currently experiencing the health state they are valuing, and valuation of described (hypothetical) health states, which is when people are asked to imagine a health state and give it a value [19, 20]. Experience-based values require the target group, for the EQ-5D-Y young people, to perform the valuation. Results have differed when comparing preferences of adults and adolescents for described health states. Prevolnik Rupel et al. found that the relative importance of different dimensions was similar [21], while Dalziel et al. [22], found differences in the Australian sample as adolescents valued the mood dimensions as worst and adults valued pain/discomfort as worst [22].

In 2020, a standardised valuation protocol for the EQ5D-Y-3L was developed to give guidance to ways in which to derive values for this youth version of the instrument to enable its use in economic evaluations [23]. According to this protocol, values for the EQ-5D-Y-3L should be elicited from an adult general population who are asked to value health states for a 10-year-old child with the TTO and DCE methods. So far, two value sets, following the guidance from the protocol [23], have been published, for Slovenia [24] and for Japan [25]. A considerable difference between how hypothetical value sets are derived for the adult version of the EQ-5D instrument [26] compared to the standardised protocol for the EQ-5D-Y-3L [23], is the applied child perspective. This difference might influence QALY weights and hence reimbursement decisions [27].

There has been an increase in studies including QALY weights for children [28] and a review of technology assessments to the National Institute for Health and Care Excellence (NICE) revealed a variety of methods being used to derive these QALY weights [29]. Clearer guidelines have been requested to help facilitate choices for decision makers [23, 30], including discussions about methods involving the state dead in the valuation task. In Sweden, for adults, the Dental and Pharmaceutical Benefits Agency (TLV) who decides on subsidy of pharmaceuticals, prefers values from persons experiencing the health state in question $[31,32]$. The view regarding whose preferences and which perspective to adopt can be considered normative $[19,20]$, but clearer guidance is warranted [30]. Previous studies indicate that values for the EQ-5D-Y-3L instrument are dependent on the choice of method, for example Kreimeier et al. [7] showed that the TTO and DCE methods yielded higher mean values for children's health states compared to adults', while the opposite was found by Kind et al. [33] using the VAS method.

Regardless of the valuation method and what perspective to use, to support researchers on how and what to report when conducting a valuation study for adults, a Checklist for Reporting Valuation studies (CREATE) has been developed 
[34]. These authors also stressed that there is no consensus in the scientific community regarding which method to use in a valuation study; hence, valuation methods are still under development. The aim of the present study was to explore EQ-5D-Y-3L experience-based VAS values derived among adolescents.

\section{Methods}

\subsection{Materials and Study Population}

EQ-5D-Y-3L and EQ VAS were included in the crosssectional population survey Life \& Health-Young People 2014, in Region Örebro, Sweden, distributed to adolescents aged 13-18 years. Åström et al. [35] have presented population data for EQ-5D-Y-3L based on the same survey, which included the EQ-5D-Y-3L Swedish version and questions on socio-demography, living conditions and health-related behaviours. There were 60-80 questions depending on the age, where older respondents also answered questions for example on alcohol use. In the current study, data are used to explore experience-based VAS values for EQ-5D-Y-3L health states. The Regional Ethical Review Board in Uppsala granted ethical permission (Dnr: 2013/459).

\subsection{The EQ-5D-Y-3L and the Visual Analogue Scale (EQ VAS)}

The EQ-5D-Y-3L is a generic HRQoL instrument, developed from the adult version of the instrument to be suitable for children and adolescents aged 8-15 years [2, 3]. The EQ-5D-Y-3L covers five dimensions of health 'mobility', 'looking after myself', 'doing usual activities', 'having pain or discomfort', and 'feeling worried, sad or unhappy', with three severity levels (no problems, some problems, a lot of problems) for each dimension. In addition to the descriptive system, the EQ-5D-Y-3L consists of the EQ VAS, where the child or adolescent rates his/her own overall health between 0 (worst) and 100 (best) imaginable health [3].

\subsection{Data Analysis}

Regression analyses were performed on individual data with VAS value as the dependent variable. The variables and the definition of models are presented in Table 1 . The VAS value was used as the given number between $0-100$ indicated by the respondent. Two dummy variables for each of the five EQ-5D-Y-3L dimensions were created representing the main effects. The dummy variable for level 2 represents the increment from level 1 (no problems) to level 2 (some problems); the dummy variable for level 3 represents the increment from level 2 (some problems) to level 3 (a lot of problems) (Table 1). We tested interaction variables that represent the occurrence of severity levels in at least one dimension which would potentially give an additional decrement to the predicted VAS values: if severity level 2 or 3 (N2); if severity level 3 (N3).

Ordinary least-squares (OLS) regressions were estimated with the main effects (Model 1); subsequently the interaction variables were entered with the N2 being statistically significant (Model 2). In all OLS models robust Standard Errors

Table 1 Definition of variables and models

\begin{tabular}{|c|c|}
\hline Variable & Definition \\
\hline MOB2 & 1 if mobility at level 2 or $3 ; 0$ otherwise \\
\hline MOB3 & 1 if mobility at level $3 ; 0$ otherwise \\
\hline LAM2 & 1 if looking after myself at level 2 or $3 ; 0$ otherwise \\
\hline LAM3 & 1 if looking after myself at level 3; 0 otherwise \\
\hline DUA2 & 1 if doing usual activities at level 2 or $3 ; 0$ otherwise \\
\hline DUA3 & 1 if doing usual activities at level 3; 0 otherwise \\
\hline HPD2 & 1 if having pain or discomfort at level 2 or $3 ; 0$ otherwise \\
\hline HPD3 & 1 if having pain or discomfort at level 3; 0 otherwise \\
\hline FWSU2 & 1 if feeling worried sad or unhappy at level 2 or $3 ; 0$ otherwise \\
\hline FWSU3 & 1 if feeling worried sad or unhappy at level 3; 0 otherwise \\
\hline $\mathrm{N} 2$ & 1 if any dimension at level 2 or $3 ; 0$ otherwise \\
\hline OLS models on EQ-5D-Y-3L & Functions \\
\hline Model 1 & f (MOB2 MOB3 LAM2 LAM3 DUA2 DUA3 HPD2 HPD3 FWSU2 FWSU3) \\
\hline Model 2 & f (MOB2 MOB3 LAM2 LAM3 DUA2 DUA3 HPD2 HPD3 FWSU2 FWSU3 N2) \\
\hline GLMs on EQ-5D-Y-3L & Functions \\
\hline Model 3 & f (MOB2 MOB3 LAM2 LAM3 DUA2 DUA3 HPD2 HPD3 FWSU2 FWSU3) \\
\hline Model 4 & f (MOB2 MOB3 LAM2 LAM3 DUA2 DUA3 HPD2 HPD3 FWSU2 FWSU3 N2) \\
\hline
\end{tabular}

$G L M$ generalized linear models, $O L S$ ordinary least squares 
(SE) were reported because of potential heteroscedasticity. In addition to OLS, generalized linear models (GLMs) were tested with a binomial distribution: Model 3 with the main effects and Model 4 with the significant N2 term. The GLMs were tested as they were shown to provide better predictive performance than OLS in the creation of experience-based VAS value sets for EQ-5D-3L and EQ-5D-5L in Germany $[12,13]$, and tested in the development of an experiencebased VAS value set for EQ-5D-5L in Sweden [14]. The N3 variable was not statistically significant in either the GLMs or the OLS models (results not shown).

To assess the different models the primary consideration was to confirm that the decrement in VAS value increased as problems reported in the different dimensions increased, known as monotonic consistency. Model 1 and Model 2 were further investigated by comparing the goodness of fit of the models. Regarding GLM, all coefficients in Model 3 and Model 4, except the coefficients for LAM3 and DUA3, were statistically significant and these models were also considered for further investigation. Predicted VAS values were compared with the observed VAS values by calculation of mean absolute error (MAE), root mean square error (RMSE) and Spearman's rank correlation coefficients, where higher correlations and lower MAE and RMSE indicate a better model fit [36]. MAE, RMSE and Spearman's rank correlation were investigated including health states reported by more than five and ten respondents, respectively. For the OLS models, adjusted $\mathrm{R}^{2}$ was assessed with higher values indicating that the model explains a larger percentage of the variation in the dependent variable, i.e., the VAS value. For the GLM models, Akaike information criterion (AIC) and Bayesian information criterion (BIC) were considered to assess the quality of models, where lower values indicated a better model fit [36].

Making the choice of the final model to use for the calculation of the VAS values for the EQ-5D-Y-3L, the criteria of monotonicity, goodness of fit, simplicity of the model and understandability were considered [37]. A split sample, randomly divided $(n=3,216)$, validation was performed to test the robustness of Model 1.

The VAS is anchored between 0 (worst) and 100 (best) imaginable health, which did not allow for anchoring between the 0 (dead) and 1 (full health) scale, a requirement for QALY calculations. One of many possible approaches to anchor the VAS values predicted by Model 1 at 'dead' and full health was explored by anchoring 0 at the predicted value for the worst health state in the EQ-5D-Y-3L descriptive system, i.e., 33333 and 100 at the predicted value for the best health state, i.e., 11111 [16]. A rescaled value for each health state $(\mathrm{h})$ can be derived using the following formula: $\left(\right.$ Rating $_{\mathrm{h}}-$ Rating $\left._{33333}\right) /\left(\right.$ Rating $_{11111}-$ Rating $_{33333}$ ) [17]. Statistical analyses were performed using SAS version 9.4, using a 5\% significance level.

\section{Results}

The response rate for the survey was $79.7 \%(n=7,399)$. Respondents who had missing or ambiguous answers for sex $(1.6 \%)$, age $(0.9 \%)$, any of the EQ-5D-Y-3L dimensions (3.4\%) or the EQ VAS (1.6\%) were excluded, which resulted in a final sample of 6,468 participants. Respondents' mean age was 15.9 years and girls made up half of the sample (49.5\%) (Table 2). In total, 92 health states were reported from the total 243 possible health states. Mean EQ VAS score was 75.4. Most problems were reported in the mood dimensions, where $4.5 \%$ and $32.2 \%$ reported "a lot of" and "some" problems, followed by 'pain/discomfort' where $2.8 \%$ and $35.8 \%$ reported a lot of and some problems. With 'doing usual activities', $0.8 \%$ and $8.1 \%$ reported a lot of and some problems. Less than $5 \%$ reported some or a lot of problems in the dimensions 'mobility' and 'looking after myself'.

The results from the OLS regression analysis, with robust SE, are presented in Table 3. In both OLS models, monotonicity was observed, namely a logical decrement in VAS value, with a decrement moving from level 1 to level 2 and additional decrement moving from level 2 to 3 for all dimensions. The largest decrements were observed in the dimension 'feeling worried, sad or unhappy', in Model 1, the coefficient for moving from level 1 to level 2 was 11.22, in absolute terms, and 13.32 for moving from level 2 to 3 . The second largest decrements were found in the dimension 'doing usual activities', where in Model 1 the coefficient for moving from level 1 to 2 was 9.39. In Model 1, the coefficients for moving from level 2 to 3 in the dimensions 'mobility', 'looking after myself' and 'doing usual activities' were not statistically significant. The N2 variable added to Model 2 resulted in a statistically significant coefficient of 1.70. For Model 1 and Model 2, the adjusted $R^{2}$ were the same (0.284) (Table 3). Similar pattern as for the OLS models were observed for the GLMs, with largest decrements observed in the dimension 'feeling worried, sad or unhappy'. The coefficients for the N2 variable were statistically significant in Model 4. The second largest decrement was the coefficient representing moving from no problems to some problems (from level 1 to 2 ) in the dimension 'doing usual activities'. AIC and BIC were lower in Model 4 compared to Model 3 (Table 3).

To assess the models' ability to predict VAS values, MAE, RMSE and Spearman's rank correlation were considered. Regarding strength of correlation between observed and predicted VAS values, all models, OLS and GLMs, performed similar $(r=0.50)$ (Table 4$)$. Similarly, results were also shown for all models regarding MAE and RMSE. Narrowing the analysis to include health states reported by more than five and more than ten respondents, respectively, did not result in stronger correlations or lower MAE or RMSE 
Table 2 Characteristics of respondents in the survey Life \& Healthyoung people $2014(n=6,468)$

\begin{tabular}{|c|c|c|}
\hline Variable & $\%$ & $n$ \\
\hline \multicolumn{3}{|l|}{ Sex } \\
\hline Boys & 50.5 & 3,266 \\
\hline Girls & 49.5 & 3,202 \\
\hline Mean age (years) [SD] & $15.9[1.6]$ & \\
\hline $13-14$ & 34.2 & 2,213 \\
\hline $15-16$ & 34.2 & 2,210 \\
\hline $17-18$ & 31.6 & 2,045 \\
\hline \multicolumn{3}{|l|}{ Parents' occupational status } \\
\hline One or both parents unemployed & 3.8 & 244 \\
\hline Both parents work & 73.2 & 4,737 \\
\hline Missing & 23.0 & 1,487 \\
\hline \multicolumn{3}{|l|}{ Self-rated health } \\
\hline Very good & 39.5 & 2,556 \\
\hline Good & 43.8 & 2,832 \\
\hline Neither good nor bad & 11.9 & 772 \\
\hline Bad & 3.1 & 200 \\
\hline Very bad & 1.0 & 65 \\
\hline Missing & 0.7 & 43 \\
\hline Less than good self-rated health & 16.0 & 1,037 \\
\hline \multicolumn{3}{|l|}{ Mobility } \\
\hline No problems & 95.3 & 6,167 \\
\hline Some problems & 4.3 & 277 \\
\hline A lot of problems & 0.4 & 24 \\
\hline \multicolumn{3}{|l|}{ Self-care } \\
\hline No problems & 99.0 & 6,405 \\
\hline Some problems & 0.8 & 53 \\
\hline A lot of problems & 0.2 & 10 \\
\hline \multicolumn{3}{|l|}{ Usual activities } \\
\hline No problems & 91.1 & 5,892 \\
\hline Some problems & 8.1 & 523 \\
\hline A lot of problems & 0.8 & 53 \\
\hline \multicolumn{3}{|l|}{ Pain or discomfort } \\
\hline No problems & 61.4 & 3,971 \\
\hline Some problems & 35.8 & 2,314 \\
\hline A lot of problems & 2.8 & 183 \\
\hline \multicolumn{3}{|l|}{ Worried, sad or unhappy } \\
\hline No problems & 62.3 & 4,029 \\
\hline Some problems & 33.2 & 2,149 \\
\hline A lot of problems & 4.5 & 290 \\
\hline Mean EQ VAS score [SD] & $75.4[18.0]$ & \\
\hline
\end{tabular}

$E Q$ VAS EQ-5D-Y-3L and the visual analogue scale, $S D$ standard deviation

between observed and predicted VAS values in any of the models (Table 4).

All four models performed similarly in terms of monotonicity and goodness of fit. However, in terms of simplicity and understandability, Model 1, the OLS model without dummies, was considered superior and the split validation showed that the performance of Model 1 was quite similar also with fewer participants $(n=3,216)$. A comparison between observed and predicted VAS values based on Model 1 is shown in Fig. 1.

Predicted VAS values and rescaled VAS values for all 243 EQ-5D-Y-3L health states can be found in the Online Resource Table S1. Calculation of VAS values for the EQ5D-Y-3L health states based on Model 1, with the example of how to calculate the value for the health state 22323 , is shown in Table 5, including comparison with two previous value sets $[9,10]$.

\section{Discussion}

We have explored EQ-5D-Y-3L experience-based VAS values derived from a sample of 6,468 adolescents in Sweden. Data were derived from a survey administered via schools to all adolescents in one region in Sweden. The dimension 'feeling, worried, sad or unhappy' had the strongest association with the VAS values. This is in line with previous studies in the adult general population in Sweden, generating experience-based value sets for the EQ-5D-3L and the EQ-5D-5L; the mood dimension had the strongest association with both TTO and VAS values [10, 14]. The mood dimension was also found to have the strongest association with experience-based VAS values in a previous study in China [11].

To enable summarising the five dimensions and severity levels of the EQ-5D-Y descriptive system into a single index value and investigating how the dimensions of health might impact the overall health status differently, valuation of children's and adolescents' health states is needed [5]. Before the development of the standardised valuation protocol for EQ-5D-Y-3L [23], the first value set for the EQ5D-Y-3L was derived from 10 to 11 years old children using the VAS [9]. However, the increased interest of using the EQ-5D-Y-3L in economic evaluations to assess interventions for children and adolescents have resulted in two recently developed value sets $[24,25]$. Both these value sets were derived guided by the standardised valuation protocol for EQ-5D-Y-3L [23]. The approach suggested in the protocol, i.e., to ask adults to value health states for a 10 -year-old child contrasts with our study and the study by $\mathrm{Wu}$ et al. [9], where youth themselves have valued their own health state, i.e., experience-based values were elicited. There is no consensus regarding what valuation method to use [34], and although arguments for which perspective to take have been presented in the literature [20], decision makers might not have taken a stand and maybe, there will not be a 'one fit all solution' with regard to perspective or valuation method across countries. When it comes to adults, TLV in Sweden 


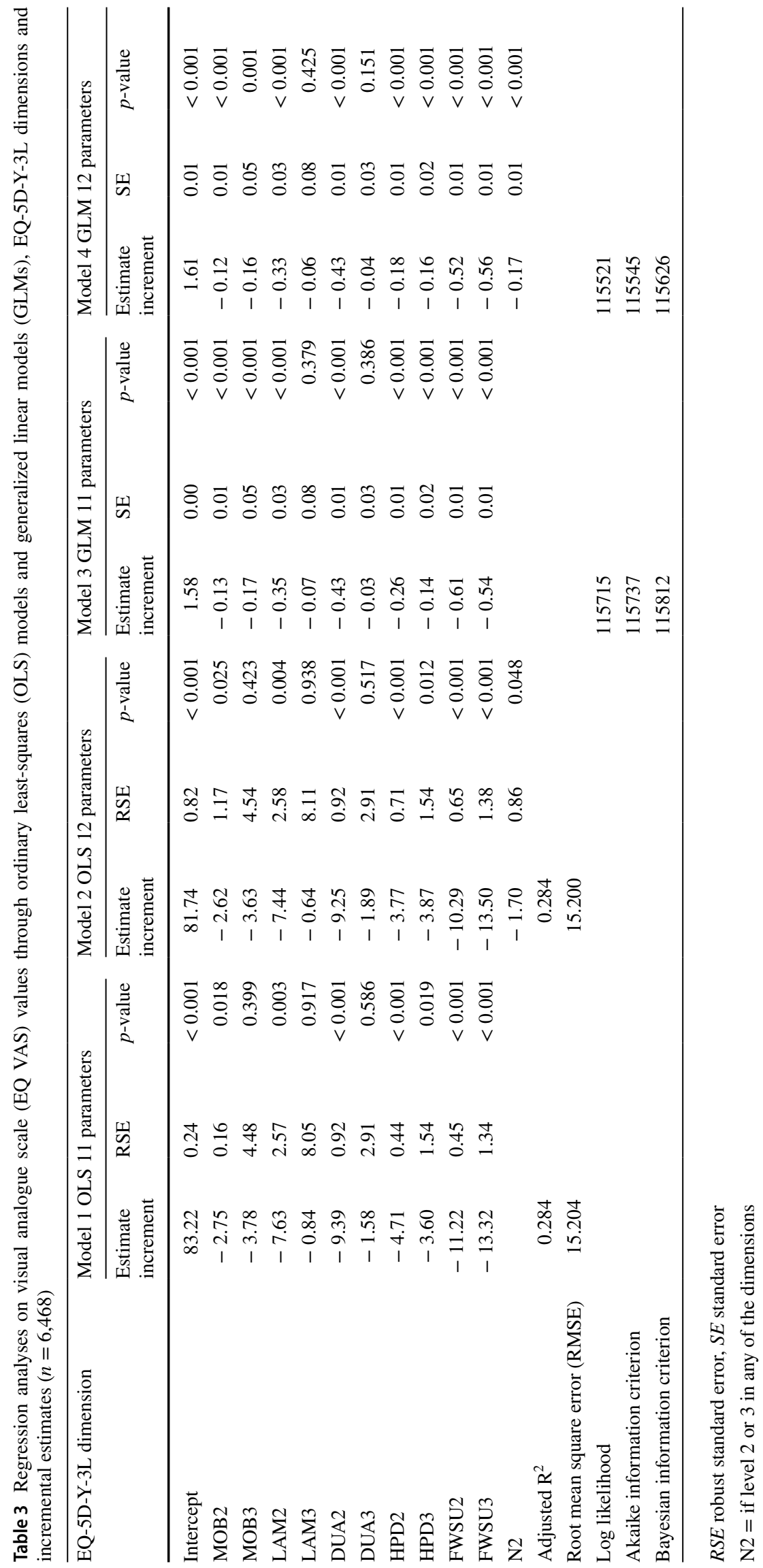


prefer values to be elicited from persons in the health condition, primarily by using SG or TTO and secondarily by using a rating scale [31, 32].

In the value sets developed for the EQ-5D-Y-3L [24, 25] the most important dimension was 'pain/discomfort' followed by the dimension 'feeling worried sad or unhappy'. In our study, second to the mood dimension the dimension 'doing usual activities' had the greatest association with the VAS value. The worst health state in the descriptive system of the EQ-5D-Y-3L is when the most severe level, a lot of problems, is reported in all dimensions, i.e., the health state 33333. In Shiroiwa et al. [25] the value was 0.29 for the health state 33333; in Prevolnik Rupel et al. [24] the value was -0.69 . In the present study the value for health state 33333 was 0.24 , and in Wu et al. 0.38 [9] (both divided by 100 to enable comparison). The negative value observed in the Slovenian value set indicates that the health state 33333 is valued as being worse than 'dead'. Generation of negative values when asking people to value described health state has been observed earlier [38]. However, a study by Bernfort et al. [39], showed that most people who lived in a health state defined by the UK value set as being worse than dead [38], did not perceive their own health state as worse than dead [39]. As value set differs regarding the range of scales, applying different value sets in economic evaluation will generate different results [27]. However, this should not limit the exploration of valuation methods and perspectives, which commonly do not yield negative values. The difference in values for health state 33333 between the study by Prevolnik Rupel et al. [24] and Shiroiwa et al. [25] is surprisingly large given that both studies are based on the standardised protocol [23]. One explanation to this could be cultural aspects [40, 41]. Differences in value sets have previously been observed, for example, when comparing value set for adults in Japan and UK, which resulted in the recommendation of a national value set for Japan [42]. It should be noted that the value for health state 33333 in the present study is much closer to the results in Shiroiwa et al. [15], even though different valuation methods and perspectives have been used in these two studies. When deriving value sets for the adult EQ-5D-3L in Slovenia, both the TTO and VAS methods were used, and substantial differences depending on valuation method were found [24]. Comparisons between value sets need to be done with caution, as there are several factors such as the valuation method, the perspective in terms of experience-based versus hypothetical health states, modelling, the age of the person valuing the health state that might influence the results [41].

An advantage with estimating experience-based values, i.e., asking people themselves to value the health states, is that we are capturing values that reflect aspects of HRQoL that are important for this age group. Hence, a strength of our study is that we have used self-reported data from 
Fig. 1 Mean observed VAS values compared to mean predicted VAS values based on Model 1 for health states with five or more observations (sorted by observed VAS value)

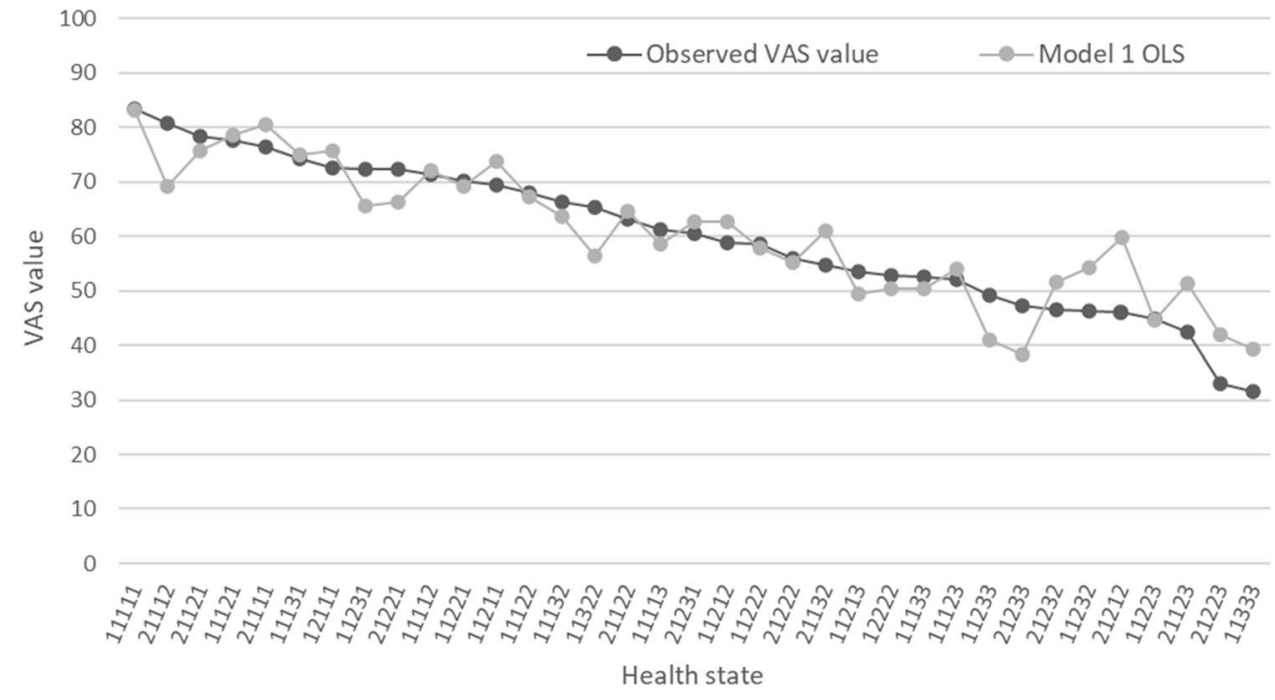

Table 5 Comparison between coefficients based on Model 1 with previous VAS value sets

\begin{tabular}{|c|c|c|c|}
\hline & $\begin{array}{l}\text { Current study } \\
\text { Model } 1^{\text {a }}\end{array}$ & $\begin{array}{l}\text { Basic model Table 3, Wu } \\
\text { et al. (2014) [9] }\end{array}$ & $\begin{array}{l}\text { Model } 4 \text { Table S4 } \\
\text { Burström et al. }(2014)^{\mathrm{b}} \\
{[10]}\end{array}$ \\
\hline Intercept & 83.22 & 85.74 & 88.86 \\
\hline \multicolumn{4}{|l|}{ Mobility } \\
\hline Level 2 & -2.75 & -2.50 & - \\
\hline Level 3 & -6.53 & -3.86 & - \\
\hline Level 2 and 3 & & - & -9.77 \\
\hline \multicolumn{4}{|c|}{ Looking after myself } \\
\hline Level 2 & -7.63 & -7.40 & - \\
\hline Level 3 & -8.47 & -10.57 & - \\
\hline Level 2 and 3 & & - & -0.79 \\
\hline \multicolumn{4}{|c|}{ Doing usual activities } \\
\hline Level 2 & -9.39 & -5.50 & -12.11 \\
\hline Level 3 & -10.98 & -10.47 & -15.00 \\
\hline \multicolumn{4}{|c|}{ Having pain or discomfort } \\
\hline Level 2 & -4.70 & -3.12 & -6.71 \\
\hline Level 3 & -8.31 & -11.28 & -12.90 \\
\hline \multicolumn{4}{|c|}{ Feeling worried, sad or unhappy } \\
\hline Level 2 & -11.22 & -4.40 & -9.96 \\
\hline Level 3 & -24.54 & -11.28 & -23.72 \\
\hline $\mathrm{N} 3$ & - & - & -9.45 \\
\hline
\end{tabular}

$V A S$ visual analogue scale

${ }^{a}$ Example of calculation of VAS value for health state 22323 (83.22-2.75-7.63-10.98-4.70-24.54= 32.62)

${ }^{\mathrm{b}}$ The adult version EQ-5D-3L was used, hence the dimensions were the following; mobility, self-care, usual activities, pain/discomfort and anxiety/depression adolescents aged 13-18 years. All adolescents in the specific age group were invited to participate, the study had a high response rate $(79.7 \%)$, and was carried out in a region with a mixed socio-economic composition; hence, we have no reason to think that adolescents enrolled in this study would differ greatly from adolescents in other regions. Therefore, it could be suggested that the results may be generalisable to Sweden as a whole. Another strength of our study is that we have used the CREATE checklist [34] as a guidance on what to report from valuation studies. Even though the checklist was developed for adults, we consider the identified elements to be equally important for valuation of children 
and adolescent health states. The guidance by the checklist enhances the reproducibility of this study. Using the TTO method to value health states for children and adolescents comes with challenges [7]. Hence, exploring the use of the VAS, as also suggested by TLV [31, 32] as an option, contributes to insights regarding valuation of health states among the younger population.

A limitation with our study was that the predicted VAS values are solely based on data from adolescents aged 13-18 years; hence, we lack information on data from the younger age group. In the study by Wu et al. [9], data were based on a sample of children aged 10-11 years and when compared to our results some differences can be observed. Another limitation was that we had no direct observation of the value for 'dead'. In a population study in China [11] the mean VAS value for 'dead' was 4.5 and the median VAS value was 0.0 . With a lack of such information in our study, instead we used the approach suggested by Sampson et al. [16], to rescale the predicted VAS values using the predicted value for the worst health state defined by the descriptive system as 'dead'. However, it might be likely that the value for 33333 would not be the same as the value for 'dead' if such data could be collected in this group. In this study we present this as one possible way of anchoring VAS values, but further research regarding ways to anchor the VAS on the 0 to 1 scale that are required for QALY calculation are needed if the VAS were be used for the purpose of economic evaluation. One suggestion could be to have adults value 'dead' using another valuation method, this having been done previously for the CHU-9D instrument [43]. The challenges with anchoring are not per se restricted to when the VAS is used as a valuation method. When latent scale DCE values are derived, as suggested in the standardised protocol [23], the DCE values need to be anchored by values elicited with the TTO method.

Using different perspectives in valuation studies among adults, valuation of experience-based versus described health states, might yield different results [44]. A different ordering of the most to the least important dimensions was seen when comparing the experience-based VAS values in the present study with the values where the general population of adults in Slovenia and Japan have valued EQ-5D-Y$3 \mathrm{~L}$ health states described to them [24, 25]. However, the ordering was the same when comparing to the Swedish experience-based TTO and VAS value sets for EQ-5D-3L [10]. The intercept, namely the VAS value for health state 11111, in both the present study and in Wu et al. [9] is lower compared to Burström et al. [10]. This indicates that young respondents rate their own overall health lower than adults on the VAS even though they report no problems across all dimensions.

\section{Conclusions}

In this study, we have explored EQ-5D-Y-3L experiencebased VAS values derived from adolescents. The dimension 'feeling worried, sad or unhappy' had the strongest association with VAS values, which is similar to what has been observed among the general adult population in Sweden when estimating value sets for both the EQ-5D-3L and EQ-5D-5L using both VAS and TTO methods. The findings suggest that it is possible for adolescents to value their own health state using the VAS, which makes it possible to capture aspects that are important for young people.

Supplementary Information The online version contains supplementary material available at https://doi.org/10.1007/s40258-021-00713-w.

Acknowledgements The authors would like to thank the adolescents for participating in the survey and Region Örebro County for providing microdata. Furthermore, discussions with Fitsum Sebsibe Teni are thankfully acknowledged. Valuable comments on earlier versions of this paper when presented in the Health Outcomes and Economic Evaluation Research Group and in the Equity and Health Policy Research Group at Karolinska Institutet are gratefully acknowledged.

Funding Open access funding provided by Karolinska Institute.

\section{Declarations}

Funding The authors received no specific funding for this work.

Conflicts of interest The authors, $\mathrm{M} \AA$ and $\mathrm{KB}$, are both members of the EuroQol Group and MA is employed by Region Stockholm. Neither of these had any influence regarding the study design, interpretation of results or in formulating the manuscript. The views expressed by the authors in the publication do not necessarily reflect the views of the EuroQol Group. The author OR declares that he has no conflict of interest.

Availability of data and material Data sharing is not possible according to Swedish law.

Code availability Not applicable.

Author contributions MÅ conceived the study and initially framed the aim of the study together with KB. The specific objectives were further developed and discussed among all authors. All authors contributed to the study design. MA cleaned the data, performed the preliminary analyses together with KB. MA interpreted initially the results. All authors contributed to the interpretation of data. MA drafted the manuscript and led the writing of the manuscript. The draft was revised for important intellectual content by KB and OR. The content of this manuscript is not under consideration for publication elsewhere and all authors have read and approved the submitted version of the manuscript, and therefore share collective responsibility and accountability for the manuscript. M $\AA$ acts as the overall guarantor.

Ethics approval The Regional Ethical Review Board in Uppsala granted ethical permission (Dnr: 2013/459). 
Consent to participate Teachers or principals at each school informed the pupils about the survey and invited them to answer the survey anonymously in the classroom during school hours. Teachers informed that participation was voluntary that they could withdraw from participation at any time. In the survey, there was written information regarding participation. To consent to participation participants were asked to put the survey in an envelope and seal it.

Consent for publication Not applicable.

Open Access This article is licensed under a Creative Commons Attribution-NonCommercial 4.0 International License, which permits any non-commercial use, sharing, adaptation, distribution and reproduction in any medium or format, as long as you give appropriate credit to the original author(s) and the source, provide a link to the Creative Commons licence, and indicate if changes were made. The images or other third party material in this article are included in the article's Creative Commons licence, unless indicated otherwise in a credit line to the material. If material is not included in the article's Creative Commons licence and your intended use is not permitted by statutory regulation or exceeds the permitted use, you will need to obtain permission directly from the copyright holder. To view a copy of this licence, visit http://creativecommons.org/licenses/by-nc/4.0/.

\section{References}

1. Devlin N, Brooks R. EQ-5D and the EuroQol group: past, present and future. Appl Health Econ Health Policy. 2017;15(2):127-37.

2. Burström K, Egmar A-C, Lugner A, Eriksson M, Svartengren M. A Swedish child-friendly pilot version of the EQ-5D instrumentthe development process. Eur J Public Health. 2010;21:171-7.

3. Wille N, Badia X, Bonsel G, Burström K, Gavrini G, Devlin N, Egmar A-C, Greiner W, Gusi N, Herdman M, Jelsma J, Kind P, Scalone L, Ravens-Sieberer U. Development of the EQ-5D-Y: a child friendly version of the EQ-5D. Qual Life Res. 2010;19(6):875-86.

4. Drummond MF, Sculpher MJ, Claxton K, Stoddart GL, Torrance GW. Methods for the economic evaluation of health care programmes. 4th ed. New York: Oxford University Press; 2015.

5. Brazier J, Ratcliffe J, Salomon AJ, Tsuchiya A. Measuring and valuing health benefits for economic evaluation. Oxford: Oxford University Press; 2007.

6. Ratcliffe J, Flynn T, Terlich F, Stevens K, Brazier J, Sawyer M. Developing adolescent-specific health state values for economic evaluation: an application of profile case best-worst scaling to the Child Health Utility 9D. Pharmacoeconomics. 2012;30(8):713-27.

7. Kreimeier S, Oppe M, Ramos-Goñi JM, Cole A, Devlin N, Herdman M, Mulhern B, Shah KK, Stolk E, Rivero-Arias O, Greiner W. Valuation of EuroQol Five-Dimensional Questionnaire, Youth Version (EQ-5D-Y) and EuroQol Five-Dimensional Questionnaire, Three-Level Version (EQ-5D-3L) Health States: The Impact of Wording and Perspective. Value Health. 2018;21(11):1291-8.

8. Kind P. Valuing EQ-5D health states-a VAStly simpler solution? In: Busschbach J, Rabin R, De Charro F, editors. 24th scientific plenary meeting of the EuroQoL group-Proceedings, $2009 \mathrm{Sept}$ 13-15; Kijkduin-The Hague, The Netherlands. Rotterdam: EuroQoL Group Executive Office; 2009. p. 319-37.

9. Wu XY, Ohinmaa A, Johnson JA, Veugelers PJ. Assessment of children's own health status using visual analogue scale and descriptive system of the EQ-5D-Y: linkage between two systems. Qual Life Res. 2014;23(2):393-402.

10. Burström K, Sun S, Gerdtham UG, Henriksson M, Johannesson M, Levin LA, Zethraeus N. Swedish experience-based value sets for EQ-5D health states. Qual Life Res. 2014;23(2):431-42.

11. Sun S, Chen J, Kind P, Xu L, Zhang Y, Burström K. Experience-based VAS values for EQ-5D-3L health states in a national general population health survey in China. Qual Life Res. 2015;24(3):693-703.

12. Leidl R, Reitmeir P. A value set for the EQ-5D based on experienced health states: development and testing for the German population. Pharmacoeconomics. 2011;29(6):521-34.

13. Leidl R, Reitmeir P. An experience-based value set for the EQ5D-5L in Germany. Value Health. 2017;20(8):1150-6.

14. Burström K, Teni FS, Gerdtham UG, Leidl R, Helgesson G, Rolfson O, Henriksson M. Experience-based Swedish TTO and VAS value sets for EQ-5D-5L Health States. Pharmacoeconomics. 2020;38(8):839-56

15. Prevolnik Rupel V, Srakar A, Rand K. Valuation of EQ-5D-3L Health States in Slovenia: VAS based and TTO based value sets. Zdr Varst. 2020;59(1):8-17.

16. Sampson C, Parkin D, Devlin N. Drop Dead: Is Anchoring at 'Dead' a Theoretical Requirement in Health State Valuation? OHE Research Paper 2020, London: Office of Health Economics. https://www.ohe.org/publications/drop-dead-anchoring-\%E2\% 80\%98dead\%E2\%80\%99-theoretical-requirement-health-statevaluation. Accessed 20 Apr 2021.

17. Shah KK, Ramos-Goñi JM, Kreimeier S, Devlin NJ. An explorations of methods for obtaining $0=$ dead anchors for latent scale EQ-5D-Y values. Eur J Heal Econ. 2020;21:1091-103.

18. Parkin D, Devlin N. Is there a case for using visual analogue scale valuations in cost-utility analysis? Health Econ. 2006;15(7):653-64.

19. Versteegh MM, Brouwer W. Patient and general public preferences for health states: a call to reconsider current guidelines. Soc Sci Med. 2016;165:66-74.

20. Helgesson G, Ernstsson O, Åström M, Burström K. Whom should we ask? A systematic literature review of the arguments regarding the most accurate source of information for valuation of health states. Qual Life Res. 2020;29(6):1465-82.

21. Prevolnik Rupel V, Ramos-Goñi JM, Ogorevc M, Kreimeier S, Ludwig K, Greiner W. Comparison of adult and adolescent preferences toward EQ-5D-Y-3L Health States. Value Health. 2021;24(9):1350-9.

22. Dalziel K, Catchpool M, García-Lorenzo B, Gorostiza I, Norman $\mathrm{R}$, Rivero-Arias O. Feasibility, validity and differences in adolescent and adult EQ-5D-Y Health State Valuation in Australia and Spain: an application of best-worst scaling. Pharmacoeconomics. 2020;38(5):499-513.

23. Ramos-Goñi JM, Oppe M, Stolk E, Shah K, Kreimeier S, RiveroArias O, Devlin N. International valuation protocol for the EQ5D-Y-3L. Pharmacoeconomics. 2020;38(7):653-63.

24. Prevolnik Rupel V, Ogorevc M, IMPACT HTA HRQoL Group. EQ-5D-y value set for Slovenia. Pharmacoeconomics. 2021;39(4):463-71.

25. Shiroiwa T, Ikeda S, Noto S, Fukuda T, Stolk E. Valuation survey of EQ-5D-Y based on the international common protocol: development of a value set in Japan. Med Decis Mak. 2021. https://doi. org/10.1177/0272989X211001859.

26. Stolk E, Ludwig K, Rand K, van Hout B, Ramos-Goñi JM. Overview, update, and lessons learned from the international EQ5D-5L valuation work: version 2 of the EQ-5D-5L Valuation Protocol. Value Health. 2019;22(1):23-30.

27. Lipman SA, Reckers-Droog VT, Kreimeier S. Think of the children: a discussion of the Rationale for and Implications of the 
Perspective Used for EQ-5D-Y Health State Valuation. Value health. 2021. https://doi.org/10.1016/j.jval.2021.01.011.

28. Kwon J, Kim SW, Ungar WJ, Tsiplova K, Madan J, Petrou S. Patterns, trends and methodological associations in the measurement and valuation of childhood health utilities. Qual Life Res. 2019;28(7):1705-24.

29. Hill H, Rowen D, Pennington B, Wong R, Wailoo A. A Review of the Methods Used to Generate Utility Values in NICE Technology Assessments for Children and Adolescents. Value Health. 2020;23(7):907-17.

30. Rowen D, Rivero-Arias O, Devlin N, Ratcliffe J. Review of valuation methods of preference-based measures of health for economic evaluation in child and adolescent populations: where are we now and where are we going? Pharmacoeconomics. 2020;38(4):325-40.

31. The Dental and Pharmaceutical Benefits Agency (TLV). General guidelines for economic evaluations from the Pharmaceutical Benefits Board LFNAR 2003:2. Stockholm: TLV; 2003. Available from: General guidelines for economic evaluations from the Pharmaceutical Benefits Board (LFNAR 2003:2) (tlv.se). Accessed 20 Apr 2021.

32. The Dental and Pharmaceutical Benefits Agency (TLV). Changes in the Dental and Pharmaceutical Benefits Agency's guidance for economic evaluations. Stockholm: TLV; 2017. Available from: https://tlv.se/download/18.467926b615d084471ac3230c/15103 16374332/TLVAR_2017_1.pdf. Accessed 20 Apr 2021.

33. Kind P, Klose K, Gusi N, Olivares P, Greiner W. Can adult weights be used to value child health states? Testing the influence of perspective in valuing EQ-5D-Y. Qual Life Res. 2015;24:2519-39.

34. Xie F, Pickard AS, Krabbe PF, Revicki D, Viney R, Devlin N, Feeny D. A Checklist for Reporting Valuation Studies of MultiAttribute Utility-Based Instruments (CREATE). Pharmacoeconomics. 2015;33(8):867-77.
35. Åström M, Persson C, Linden-Bostrom M, Rolfson O, Burström K. Population health status based on the EQ-5D-Y-3L among adolescents in Sweden: results by sociodemographic factors and self-reported comorbidity. Qual Life Res. 2018;27(11):2859-71.

36. Pham H. A new criterion for model selection. Mathematics. 2019;7:1215.

37. Williams A. The measurement and valuation of health: A chronicle. Centre for Health Economics Discussion paper 136. 1995. York: University of York.

38. Dolan P. Modeling valuations for EuroQol health states. Med Care. 1997;35(11):1095-108.

39. Bernfort L, Gerdle B, Husberg M, Levin LÅ. People in states worse than dead according to the EQ-5D UK value set: would they rather be dead? Qual Life Res. 2018;27(7):1827-33.

40. Bailey H, Kind P. Preliminary findings of an investigation into the relationship between national culture and EQ-5D value sets. Qual Life Res. 2010;19(8):1145-54.

41. Devlin N, Parkin D, Janssen B. Methods for analysing and reporting EQ-5D data. Springer International Publishing; 2020. https:// doi.org/10.1007/978-3-030-47622-9.

42. Tsuchiya A, Ikeda S, Ikegami N, Nishimura S, Sakai I, Fukuda T, Hamashima C, Hisashige A, Tamura M. Estimating an EQ-5D population value set: the case of Japan. Health Econ. 2002;11(4):341-53.

43. Chen G, Xu F. Scoring the Child Health Utility 9D instrument: estimation of a Chinese child and adolescent-specific tariff. Qual Life Res. 2019;28(1):163-76.

44. Rand-Hendriksen K, Augestad LA, Kristiansen IS, Stavem K. Comparison of hypothetical and experienced EQ-5D valuations: relative weights of the five dimensions. Qual Life Res. 2012;21(6):1005-12. 